H. YAMADA

KODAI MATH. J.

3 (1980), 341-357

\title{
ON THE FIRST BOUNDARY VALUE PROBLEMS FOR SOME DEGENERATE SECOND ORDER ELLIPTIC DIFFERENTIAL EQUATIONS
}

\author{
BY HARUKI YAMADA
}

\section{$\S 1$. Introduction.}

Let $\Omega \subset \boldsymbol{R}^{n}$ be an bounded open domain with $C^{\infty}$-boundary $\Sigma=\partial \Omega$. We consider the following so called "first boundary value problem".

$$
\left\{\begin{array}{l}
L(u) \equiv\left(a^{k j} u_{x_{k}}\right)_{x_{j}}+b^{k} u_{x_{k}}+c u=f \quad \text { in } \Omega, \\
\left.u\right|_{\Sigma}=0,
\end{array}\right.
$$

where $u_{x_{k}}=\partial u / \partial x_{k}$, etc., and the summation convention such as $\left(a^{k \jmath} u_{x_{k}}\right)_{x_{\jmath}}$ $=\sum_{k, j=1}^{n}\left(a^{k j} u_{x_{k}}\right)_{x}$, are used. Without loss of generality, we can assume that $a^{k \jmath}=a^{j k}$ (see the following condition [A.1]). Throughout this paper, we pose the following assumptions:

$$
a^{k \jmath}, b^{k}, c, f \in C^{\infty}(\bar{\Omega}) \text { and real valued. }
$$

$$
a^{k j} \xi_{k} \xi_{j} \geqq 0 \quad \text { for all }(x, \xi) \in \bar{\Omega} \times \boldsymbol{R}^{n} \text {. }
$$

$$
c<0, \quad c-b_{x_{k}}^{k}<0 \quad \text { on } \bar{\Omega} \text {. }
$$

$$
a^{k j} \xi_{k} \xi_{\jmath}>0 \quad \text { for all }(x, \xi) \in \Sigma \times\left(\boldsymbol{R}^{n} \backslash 0\right) .
$$

Under some additional assumptions for the boundary behavior of the coefficients replaced by [A.4], Kohn-Nirenberg [5], [6] and Oleinik [7], [8] proved several existence, uniqueness and regularity theorems for the problem $[\mathrm{P}]$. For example, from their results we have the following theorem. (see e.g. [8] Chap. I, § 5).

THEOREM 1.1. If in addition to [A.1], [A.2], [A.3], we assume that

$$
a^{k J} \nu_{k} \nu_{\jmath}>0 \quad \text { for all } x \in \Sigma \text {, }
$$

where $\nu=\left(\nu_{1}, \cdots, \nu_{n}\right)$ is the unit normal vector for $\Sigma$ at $x$, then for any $f \in C^{\infty}(\bar{\Omega})$, there is a uniquely determined weak solution $u \in L^{2}(\Omega)$ of $[\mathrm{P}]$. Moreover, there

Received June 5, 1979 
is a constant $C$ independent on $f$ such that

$$
\|u\|_{0, \Omega} \leqq C\|f\|_{0, \Omega} .
$$

Here the weak solution of $[\mathrm{P}]$ is defined by the following manner: Let $L^{*}$ be the adjoint of $L$, i. e.

Then

$$
L^{*}(v) \equiv\left(a^{k} v v_{x_{j}}\right)_{x_{k}}-\left(b^{k} v\right)_{x_{k}}+c v
$$

Definition 1.2. $u \in L^{2}(\Omega)$ is called the weak solution of $[\mathrm{P}]$ if

$$
\int_{\Omega} u L^{*}(v) d x=\int_{\Omega} v f d x
$$

is verified for all $v \in C^{\infty}(\bar{\Omega})$ with $\left.v\right|_{\Sigma}=0$.

Of course the weak solution equals the classical (strong) solution if $u \in$ $C^{2}(\Omega) \cap C^{0}(\bar{\Omega})$.

The solution whose existence is guaranteed in Theorem 1.1 is known as the weak limit of the solutions $u_{\varepsilon} \in C^{\infty}(\bar{\Omega})$ as $\varepsilon \rightarrow+0$ of the following approximate elliptic boundary value problems $\left[\mathrm{P}_{\varepsilon}\right]$ :

$$
\left\{\begin{array}{l}
L_{\varepsilon}(u) \equiv L(u)+\varepsilon \Delta u=f \quad \text { in } \Omega, \\
\left.u\right|_{\Sigma}=0 .
\end{array}\right.
$$

Several facts are known concerning the regularity of the weak solution $u \in L^{2}(\Omega)$ of [P] (see especially [6], [8]). Among those results it is remarkable that; in order to assure that $u \in C^{m}(\bar{\Omega}), c$ must sufficiently be small (i.e. $c \leqq-c_{0}$ on $\bar{\Omega}$ for sufficiently large $c_{0}>0$ ) and the modulus $c_{0}$ of the smoothness are determined by $m$ and the values of the derivatives of $a^{k \jmath}$ (resp. $b^{k}$ ) up to order 2 (resp. 1). Especially, in general, $c_{0}$ is an increasing function of $m$ and we cannot assert $u \in C^{\infty}(\bar{\Omega})$ for finite $c_{0}$.

ExAmPLE. Let $\Omega=\left\{x \in \boldsymbol{R}^{n} ;|x|=1\right\}$ and let $L$ be given by

$$
L u \equiv|x|^{2} \Delta u+c u=0 .
$$

By a result of [8] it is easy to see that for any $m \in N$ there is a constant $c_{0}=$ $c_{0}(m)$ such that

$$
\text { if } c \leqq-c_{0} \text { on } \bar{\Omega} \text {, then the weak solution } u \in L^{2}(\Omega) \text { is in } C^{m}(\bar{\Omega}) \text {. }
$$

But there are no $c_{0}>0$ for which (1.1) is true for $m=\infty$. In fact, if we take $u=|x|^{\alpha}-1(\alpha>0)$, it is clear that $u$ is the weak solution of $[\mathrm{P}]$ for $c=-\alpha(\alpha-2)$ $-\alpha n$. Note that $u \notin C^{[\alpha]+1}(\bar{\Omega})$ unless $\alpha \in N$. 
The aim of this paper is to find a sufficient condition for the weak solution to be $u \in C^{\infty}(\bar{\Omega})$. In doing so, we use the $L^{2}$-method which was used in [5] rather than the maximum principle method used in [7]. Our main result is stated in $\S 3$ as Theorem 3.1.

In $\S 2$ we shall prove some estimates for the solutions $u_{\varepsilon} \in C^{\infty}(\bar{\Omega})$ of the approximate problems $\left[\mathrm{P}_{\varepsilon}\right]$. In $\S 3$, by using those estimates, we shall give a sufficient condition for $u \in C^{\infty}(\bar{\Omega})$. In $\S 4$, as applications, we show examples of smoothness and non-smoothness of solutions of some simple boundary value problems. The relations between regularity of solutions of boundary value problems and hypoellipticity of operators will also be noted. Our examples contain a case where the operator is not hypoelliptic but all the solutions of the boundary value problem belong to $C^{\infty}(\bar{\Omega})$.

Our assumption [A.4] in Theorem 3.1 seems to be too restrictive. In fact, as will be indicated in $\S 4$, without [A.4], one will be able to prove regularities of solutions of the boundary value problem on a certain set containing the neighbourhood of $\Sigma_{2}$ and $\Sigma_{3}$ as long as we assume that $\Sigma_{2}, \Sigma_{3}$ and $\Sigma_{0} \cup \Sigma_{1}$ are disjoint. (For definitions of $\Sigma_{0}, \cdots, \Sigma_{3}$ see e.g. [8]). One powerful method for the proof of this fact will be the method of the stochastic differential equations (probability methods). In this connection, we refer to [2], [3] and [9]. In this paper, however, we have used only the $L^{2}$-method. Hence our condition (3.2) for the regularity of solutions may only be interesting on the set where all the vector fields $X_{0}, X_{1}, \cdots, X_{n}$ degenerate. (For the definition of $X_{0}, \cdots, X_{n}$ and the relations between $X_{0}, \cdots, X_{n}$ and the regularity problem see [4], [8] and $\S 4$ of this paper).

I would like to express my sincere gratitude to professor Yoshikazu Hirasawa for his encouragements and advices.

\section{$\S 2$. Inequalities.}

In this section we shall prove some basic inequalities by using mainly the technique used in [5]. Though, in [5], [6], it is proved that for any $m \in N$, there is a sufficiently large constant $c_{0}>0$ such that if $c \leqq-c_{0}$ on $\bar{\Omega}$, then $[\mathrm{P}]$ has a unique solution $u \in C^{m}(\bar{\Omega})$, the modes of dependence of $c_{0}$ with respect to $a^{k \jmath}, b^{k}$ are not clarified. Especially, it is not possible to decide whether the solution belongs to $C^{\infty}(\bar{\Omega})$ or not. We shall somewhat clarify this situation by accurate estimates. But in doing so, we have to pose rather strong restriction [A.4] for the behavior of the coefficients $a^{k j}$ near the boundary.

We shall use the following standard notations:

(*)

$$
\begin{aligned}
\|\mathfrak{u}\|_{0, \Omega}^{2} & =\int_{\Omega} \mathfrak{l} \cdot \overline{\mathfrak{u}} d x, \quad(\mathfrak{l}, \mathfrak{v})=(\mathfrak{u}, \mathfrak{v})_{\Omega}=\int_{\Omega} \mathfrak{l} \cdot \overline{\mathfrak{v}} d x, \\
\|\mathfrak{l}\|_{\mu, \Omega}^{2} & =\sum_{|\alpha| \leqq \mu}\left\|\partial^{\alpha} \mathfrak{u}\right\|_{0, \Omega}^{2},
\end{aligned}
$$


where $\partial^{\alpha}=\partial_{1}^{\alpha_{1}} \cdots \partial_{n}^{\alpha_{n}}, \partial_{j}=\partial / \partial x_{\jmath}$. If we write

we have

$$
X_{j}=a^{k j} \partial_{k}, \quad X_{0}=b^{k} \partial_{k},
$$

$$
L_{\varepsilon}(u)=\varepsilon \Delta u+\partial_{j} X_{j} u+X_{0} u+c u .
$$

As in [5], we introduce the following bilinear form $Q_{\varepsilon}$ associated with $L_{\varepsilon}$ :

$$
Q_{\varepsilon}(\mathfrak{u}, \mathfrak{v}) \equiv \int_{\Omega}\left\{\varepsilon \partial_{j} \mathfrak{u} \cdot \partial_{j} \overline{\mathfrak{y}}+X_{j} \mathfrak{u} \cdot \partial_{j} \overline{\mathfrak{y}}+\frac{1}{2}\left(\mathfrak{u} \cdot X_{0} \overline{\mathfrak{y}}-X_{0} \mathfrak{u} \cdot \overline{\mathfrak{v}}\right)-\frac{1}{2}\left(2 c-b_{x_{j}}^{\mathfrak{j}}\right) \mathfrak{u} \cdot \overline{\mathfrak{v}}\right\} d x .
$$

Note that when $\mathfrak{u}, \mathfrak{v} \in C^{\infty}(\bar{\Omega}),\left.\mathfrak{u}\right|_{\Sigma}=0,\left.\mathfrak{v}\right|_{\Sigma}=0$, then by partial integrations, we have

$$
Q_{\varepsilon}(\mathfrak{u}, \mathfrak{b})=-\left(L_{\varepsilon}(\mathfrak{u}), \mathfrak{v}\right) .
$$

Remark 1. In what follows, all the functions $u, u_{\varepsilon}$ which appear as solutions of the boundary value problems are real valued. But since, in $\S 2$, we have to consider the functions $A u_{\varepsilon}$ where $A$ is a differential operator with a complex coefficient, we use the above notation $\left(^{*}\right)$ instead of

$$
(u, v)=\int_{\Omega} u \cdot v d x .
$$

In the followings, when we use the roman letters $u, u_{\varepsilon}$ to represent functions, it always means that they are real valued functions.

By [A.2] and [A.3], we have

$$
C_{1}\|u\|_{0}^{2} \leqq\left(-\frac{1}{2}\left(2 c-b_{x_{j}}^{\jmath}\right) u, u\right) \leqq Q_{\varepsilon}(u, u) \quad \text { for any } u \in C^{\infty}(\bar{\Omega}),
$$

where $C_{1}$ is a positive constant. Further, if there are an open set $U \subset \Omega$, some positive constant $C_{2}$ and some $m, 0 \leqq m \leqq n-1$, such that

$$
a^{k j} \xi_{k} \xi_{j} \geqq C_{2} \sum_{j=m+1}^{n} \xi_{j}^{2} \quad \text { for all }(x, \xi) \in \bar{U} \times \boldsymbol{R}^{n},
$$

then we have

$$
\begin{gathered}
C_{2} \sum_{\jmath=m+1}^{n}\left\|\partial_{j} u\right\|_{0, U}^{2}+\left(-\frac{1}{2}\left(2 c-b_{x_{j}}^{\jmath}\right) u, u\right) \leqq Q_{\varepsilon}(u, u) \\
\text { for all } u \in C^{\infty}(\bar{\Omega}) .
\end{gathered}
$$

Note that if $L$ is elliptic on $\bar{U}$ (i.e. $m=0$ ), then

$$
C_{2}\|u\|_{1, U}^{2} \leqq Q_{\varepsilon}(u, u) \quad \text { for all } u \in C^{\infty}(\bar{\Omega}) .
$$

Remark 2. If $m=n$, the condition (2.4) is meaningless. Then, by the condition (2.4), we shall understand that we do not impose any conditions at all. 
When we estimate $\|u\|_{\mu, \Omega}$, it is convenient to introduce the following operators:

$$
A=\varphi D^{\alpha}, \quad A^{*}=D^{\alpha} \cdot \varphi \cdot=\varphi D^{\alpha}+\sum_{j \in \alpha} D_{j} \varphi \cdot D^{\alpha-\jmath}+\cdots
$$

where $\varphi$ is a real valued function in $C_{0}^{\infty}(\Omega)$ and $D^{\alpha}=D_{1}^{\alpha_{1}} \cdots D_{n}^{\alpha_{n}}, D_{j}=1 / \imath \partial / \partial x_{\jmath}$. Further we have used the following abbreviations:

$$
D^{\alpha-\jmath}=D_{1}^{\alpha_{1}} \cdots D_{j}^{\alpha_{j}-1} \cdots D_{n}^{\alpha_{n}},
$$

and the sum $\sum_{j \in \alpha}$ means that $j$ are taken from all possible indices which appear in $\alpha=\left(\alpha_{1}, \cdots, \alpha_{n}\right)$ counting its multiplicities $\alpha_{\jmath}$. Accordingly we have

$$
\sum_{j \in \alpha} D_{j} \varphi \cdot D^{\alpha-\jmath}=\sum_{j=1}^{n} \alpha_{j} D_{j} \varphi \cdot D_{1}^{\alpha_{1}} \cdots D_{\jmath}^{\alpha_{j}-1} \cdots D_{n}^{\alpha_{n}} .
$$

In Lemma 2.5, it will also be used the following abbreviations:

$$
D^{\alpha-j+k}=D^{\alpha-\jmath} D_{k}=D_{1}^{\alpha_{1}} \cdots D_{\jmath}^{\alpha_{j}-1} \cdots D_{k}^{\alpha_{k}+1} \cdots D_{n}^{\alpha_{n}} .
$$

LEMMA 2.1 (c.f. [8]). Let $M$ and $N$ be differential operators of order not higher than 1 with real coefficients. Then for any $u \in C^{\infty}(\bar{\Omega})$, we have

$$
\begin{aligned}
2 & \left\{(M A u, N A u)-\left(M u, N A^{*} A u\right)\right\} \\
= & 2([M, A] u,[N, A] u)+\left(\left(A-A^{*}\right) M u,[N, A] u\right)+\left(M u,\left[N, A-A^{*}\right] A u\right) \\
& -(M u,[[N, A], A] u)+\left(\left(A-A^{*}\right) N u,[M, A] u\right)+\left(N u,\left[M, A-A^{*}\right] A u\right) \\
& -(N u,[[M, A], A] u)+\left(M A^{*} A u, N u\right)-\left(M u, N A^{*} A u\right),
\end{aligned}
$$

where by definition $[X, Y]=X Y-Y X$ for any two operators $X$ and $Y$.

Proof. First note that by definition and partial integrations,

$$
(A u, v)=\left(u, A^{*} v\right) .
$$

By using further partial integrations,

$$
\begin{aligned}
& 2\left\{(M A u, N A u)-\left(M u, N A^{*} A u\right)\right\} \\
&=(M A u, N A u)-\left(M u, N A^{*} A u\right)+(M A u, N A u) \\
&-\left(M A^{*} A u, N u\right)+\left(M A^{*} A u, N u\right)-\left(M u, N A^{*} A u\right) \\
&=([M, A] u, N A u)-\left(M u,\left[N, A^{*}\right] A u\right)+(M A u,[N, A] u) \\
&-\left(\left[M, A^{*}\right] A u, N u\right)+\left(M A^{*} A u, N u\right)-\left(M u, N A^{*} A u\right) .
\end{aligned}
$$

From the second and the third term in the right hand side, we have 


$$
\begin{aligned}
(M A u, & {[N, A] u)-\left(M u,\left[N, A^{*}\right] A u\right) } \\
= & ([M, A] u,[N, A] u)+\left(\left(A-A^{*}\right) M u,[N, A] u\right) \\
& +\left(M u,\left[N, A-A^{*}\right] A u\right)-(M u,[[N, A], A] u) .
\end{aligned}
$$

From the first and the fourth term in the right we have a similar formula. Combining these formulas we have (2.6).

LEMMA 2.2. Let (2.4) be true for some $m, 0 \leqq m \leqq n-1$ and some $U \subset \Omega$. Then for any $\alpha,|\alpha|=\mu, \varphi \in C_{0}^{\infty}(U)$ and solutions $u=u_{\varepsilon} \in C^{\infty}(\bar{\Omega})$ of $\left[\mathrm{P}_{\varepsilon}\right]$, there is a constant $C>0$ such that

$$
\sum_{\jmath=m+1}^{n}\left\|\varphi \partial^{\alpha} \partial_{j} u\right\|_{0, \Omega}^{2} \leqq C\left(\|f\|_{\mu, U}^{2}+\|u\|_{\mu, U}^{2}\right) .
$$

Proof. By (2.5), it is sufficient to prove that

$$
\left|Q_{\varepsilon}\left(\varphi \partial^{\alpha} u, \varphi \partial^{\alpha} u\right)\right| \leqq C\left(\|f\|_{\mu, U}^{2}+\|u\|_{\mu, U}^{2}\right) .
$$

Let $A=\varphi D^{\alpha}$. Then $Q_{\varepsilon}\left(\varphi \partial^{\alpha} u, \varphi \partial^{\alpha} u\right)=Q_{\varepsilon}(A u, A u)$ and

$$
\begin{aligned}
Q_{\varepsilon}(A u, A u) & =Q_{\varepsilon}(A u, A u)-Q_{\varepsilon}\left(u, A^{*} A u\right)+Q_{\varepsilon}\left(u, A^{*} A u\right) \\
& =Q_{\varepsilon}(A u, A u)-Q_{\varepsilon}\left(u, A^{*} A u\right)-\left(f, A^{*} A u\right) .
\end{aligned}
$$

Note that

$$
\left|\left(f, A^{*} A u\right)\right|=|(A f, A u)| \leqq \frac{1}{2}\left(\|f\|_{\mu, U}^{2}+\|u\|_{\mu, U}^{2}\right) .
$$

Next, we have to estimate the term

$$
Q_{\varepsilon}(A u, A u)-Q_{\varepsilon}\left(u, A^{*} A u\right) .
$$

This term can be decomposed into the several terms of the form

$$
(M A u, N A u)-\left(M u, N A^{*} A u\right)
$$

according to (2.1). Here the possible pairs of $M$ and $N$ are the followings:

$$
\begin{aligned}
& M=N=\partial_{\jmath} ; \quad M=X_{\jmath}, N=\partial_{\jmath} ; \quad M=X_{0}, N=1 ; \\
& M=1, N=X_{0} ; \quad M=\left(2 c-b_{x_{k}}^{k}, N=1 ; \quad j=1,2, \cdots, n .\right.
\end{aligned}
$$

Thus it is enough to estimate these terms of the form (2.8) appearing in (2.7).

If we note that $A=\varphi D^{\alpha},|\alpha|=\mu, \varphi \in C_{0}^{\infty}(U)$ and the fact that $M, N$ are operators of order not higher than 1 , we have, by Lemma 2.1,

$$
\begin{aligned}
& \left|(M A u, N A u)-\left(M u, N A^{*} A u\right)\right| \\
& \quad \leqq C\|u\|_{\mu, U}^{2}+\frac{1}{2}\left|\left(M A^{*} A u, N u\right)-\left(M u, N A^{*} A u\right)\right| .
\end{aligned}
$$


In fact, in (2.6), each terms except the term $\left(M A^{*} A u, N u\right)-\left(M u, N A^{*} A u\right)$ can be estimated by $C\|u\|_{\mu, U}^{2}$ by means of partial integration. Further, if we note $a^{k \jmath}=a^{j k}$, we have that, in (2.7), the term of the form $\left(M A^{*} A u, N u\right)-\left(M u, N A^{*} A u\right)$ may only appear when $M=1, N=X_{0}$ and $M=X_{0}, N=1$. But then, we have

$$
\left|\left(X_{0} u, A^{*} A u\right)-\left(u, X_{0} A^{*} A u\right)\right| \leqq\left|\left(\left[A^{*}, X_{0}\right] A u, u\right)\right|+\left|\left(\left[A, X_{0}\right] u, A u\right)\right|
$$

This proves the lemma.

$$
\leqq C\|u\|_{\mu, U}^{2} \cdot
$$

Corollary 2.3. Let $\alpha=\left(\alpha_{1}, \cdots, \alpha_{m}, \alpha_{m+1}, \cdots, \alpha_{n}\right), \nu=\sum_{k=1}^{m} \alpha_{k}$. Then, for the solution $u=u_{\varepsilon} \in C^{\infty}(\bar{\Omega})$ of $\left[\mathrm{P}_{\varepsilon}\right]$, we have

$$
\left\|\varphi \partial^{\alpha} u\right\|_{0, \Omega}^{2} \leqq C\left(\|f\|_{\mu, U}^{2}+\|u\|_{\nu, U}^{2}\right) .
$$

Especially, if (2.4) is valid for $m=0$, then

$$
\left\|\varphi \partial^{\alpha} u\right\|_{0, \Omega}^{2} \leqq C\|f\|_{\mu, U}^{2} .
$$

Proof. Easy by Lemma 2.2 and the induction arguments.

The following lemma, which is trivial from Corollary 2.3 when $\bar{V} \Subset \Omega$, is a well known fact in the theory of elliptic boundary value problems and we shall omit the proof. (see e.g. [1] $\S 9$ ).

LEMMA 2.4. Let $V \subset \Omega$ be an open subset (we may allow the case when $\bar{V} \cap \Sigma$ $\neq \emptyset)$. Suppose $L$ is elliptic on $\bar{V}$. Then for the solution $u=u_{\varepsilon} \in C^{\infty}(\bar{\Omega})$ of $\left[\mathrm{P}_{\varepsilon}\right]$, we have

$$
\|u\|_{\mu, v}^{2} \leqq C\|f\|_{\mu, \Omega}^{2},
$$

where $C$ is independent on $\varepsilon$.

Let $\omega \subset \Omega$ be the maximal set on which $L$ is not elliptic. In what follows, we shall call this set the non-elliptic set of $L$ in $\Omega$. By the assumption [A.4], we can take a sufficiently small neighbourhood $\hat{\omega}$ of $\omega$ such that $\hat{\omega} \cap \Sigma=\emptyset$.

LEMMA 2.5. Suppose that (2.4) is valid for some $m, 0 \leqq m \leqq n$, and for $U=\Omega$. Then for any fixed (small) $\delta>0$, any $\alpha=\left(\alpha_{1}, \cdots, \alpha_{m}, 0, \cdots, 0\right),|\alpha|=\mu$, and solutrons $u=u_{\varepsilon} \in C^{\infty}(\bar{\Omega})$ of $\left[P_{\varepsilon}\right]$, we have the following inequality.

$$
\begin{aligned}
\left(-\frac{1}{2}\left(2 c-b_{x_{j}}^{j}\right) \partial^{\alpha} u, \partial^{\alpha} u\right)_{\hat{\omega}} \leqq & \delta \sum_{\beta=\left(\beta_{1}, \cdots, \beta m, 0, \cdots, 0\right)}\left\|\partial^{\beta} u\right\|_{0, \hat{\omega}}^{2} \\
& +\sum_{k=1}^{m} \sum_{s \in \alpha}\left(b_{x_{s}}^{k} \partial^{\alpha-s+k} u, \partial^{\alpha} u\right)_{\hat{\omega}}
\end{aligned}
$$




$$
\begin{aligned}
& +\frac{1}{2} \sum_{k, j=1}^{m} \sum_{s, l \in \alpha}\left(a_{x_{s} x_{l}}^{k \jmath} \partial^{\alpha-l+\jmath} u, \partial^{\alpha-s+k} u\right)_{\hat{\omega}} \\
& +C\left(\|f\|_{\mu, \Omega}^{2}+\|u\|_{\mu-1, \Omega}^{2}\right),
\end{aligned}
$$

where the constant $C>0$ is independent on $\varepsilon$.

Remark 3. For the meaning of the condition of the above lemma when $m=n$, see Remark 2 of this section.

Proof. Take and fix a real valued function $\varphi \in C_{0}^{\infty}(\Omega)$ with $\varphi \equiv 1$ on $\hat{\omega}$ and $\varphi \geqq 0$ on $\Omega$. Then from (2.3),

$$
\begin{aligned}
\left(-\frac{1}{2}\left(2 c-b_{x_{j}}^{j_{j}}\right) \partial^{\alpha} u, \partial^{\alpha} u\right)_{\hat{\omega}} & \leqq\left(-\frac{1}{2}\left(2 c-b_{x_{j}}^{\jmath}\right) \varphi \partial^{\alpha} u, \varphi \partial^{\alpha} u\right)_{\Omega} \\
& \leqq Q_{\varepsilon}\left(\varphi \partial^{\alpha} u, \varphi \partial^{\alpha} u\right) .
\end{aligned}
$$

Let $A=\varphi D^{\alpha}$. Then $Q_{\varepsilon}\left(\varphi \partial^{\alpha} u, \varphi \partial^{\alpha} u\right)=Q_{\varepsilon}(A u, A u)$ and

$$
\begin{aligned}
Q_{\varepsilon}(A u, A u) & =Q_{\varepsilon}(A u, A u)-Q_{\varepsilon}\left(u, A^{*} A u\right)+Q_{\varepsilon}\left(u, A^{*} A u\right) \\
& =Q_{\varepsilon}(A u, A u)-Q_{\varepsilon}\left(u, A^{*} A u\right)-\left(f, A^{*} A u\right) .
\end{aligned}
$$

Note that for any $\delta>0$,

$$
\left|\left(f, A^{*} A u\right)\right|=|(A f, A u)| \leqq \frac{1}{4 \delta}\left\|\varphi \partial^{\alpha} f\right\|_{0, \Omega}^{2}+\delta\left\|\varphi \partial^{\alpha} u\right\|_{0, \Omega}^{2} .
$$

In what follows, the symbol $\delta$ generically stands for a positive constant which can be taken as small as we like. Further, by using (2.10) of Corollary 2.3, we have

$$
\left\|\varphi \partial^{\alpha} u\right\|_{0, \Omega}^{2} \leqq\left\|\partial^{\alpha} u\right\|_{0, \hat{\omega}}^{2}+C\|f\|_{\mu, \Omega}^{2} .
$$

Thus it is sufficient to estimate the term (2.7), i. e.

$$
\left\{Q_{\varepsilon}(A u, A u)-Q_{\varepsilon}\left(u, A^{*} A u\right)\right\} .
$$

This term can be decomposed into several terms of the form (2.8) where the possible pairs of $M$ and $N$ are the same as in the proof of Lemma 2.2. Thus we shall estimate each terms of the form (2.8) appearing in (2.7). Since $\varphi \equiv 1$ on $\hat{\omega}$, we have $A-A^{*}=0$ on $\hat{\omega}$. Combining this fact and Corollary 2.3, we can estimate all the terms of (2.6) containing $A-A^{*}$ by $C\left(\|f\|_{\mu, \Omega}^{2}+\|u\|_{\mu-1, \Omega}^{2}\right)$. (Integration by parts was used in obtaining these estimates). Thus it is enough to estimate the terms of the following form:

$$
\begin{aligned}
& I_{1}=([M, A] u,[N, A] u), \quad I_{2}=(M u,[[N, A], A] u), \\
& I_{3}=(N u,[[M, A], A] u), \quad I_{4}=\left(M A^{*} A u, N u\right)-\left(M u, N A^{*} A u\right) .
\end{aligned}
$$


i) Suppose $M=\partial_{\jmath}$. From $\partial_{\jmath} \varphi \equiv 0$ on $\hat{\omega}$, we have $\left[\partial_{\jmath}, A\right] u=0$ on $\hat{\omega}$. Thus

$$
\begin{aligned}
\left|I_{1}\right| & =\left|\left(\left[\partial_{\jmath}, A\right] u,[N, A] u\right)_{\Omega}\right|=\left|\left(\left[\partial_{\jmath}, A\right] u,[N, A] u\right)_{\Omega_{1} \hat{\omega}}\right| \\
& \leqq C\|f\|_{\mu, \Omega}^{2} .
\end{aligned}
$$

Here we have used the fact that $\left[\partial_{\jmath}, A\right],[N, A]$ are operators of order $|\alpha|=\mu$ and we have used Corollary 2.3. The same estimates are true when $M=\partial$, is replaced by $N=\partial_{\jmath}$. Next, in the case $N=1$ or $M=1$, it is easy to see that

$$
\left|I_{1}\right| \leqq C\|f\|_{\mu, \Omega}^{2}
$$

Thus the term $I_{1}$ is harmless.

ii) The term $I_{2}$ is also harmless. In fact, when $N=\partial$, or $N=1$, the term $[N, A] u$ vanishes on $\hat{\omega}$. Hence these terms can be estimated by $C\left(\|f\|_{\mu, \Omega}^{2}+\|u\|_{\mu-1, \Omega}^{2}\right)$. On the other hand, when $N=X_{0}$, we have $M=1$. So clearly this term can be estimated by

$$
\delta \sum_{|\beta|=\mu}\left\|\partial^{\beta} u\right\|_{0, \hat{\omega}}^{2}+\frac{C}{\delta}\|u\|_{\mu-1, \Omega}^{2}
$$

for any small fixed $\delta>0$.

iii) The term $I_{3}$ can be easily estimated by $C\left(\|f\|_{\mu, \Omega}^{2}+\|u\|_{\mu-1, \Omega}^{2}\right)$ except when $M=X_{\jmath}$ and $N=\partial_{\jmath}$. When $M=X_{\jmath}, N=\partial_{\jmath}$, we have

$$
\begin{aligned}
(N u,[[M, A], A] u) & =(-1)^{\mu}\left(\partial_{j} u,\left[\left[a^{k j} \partial_{k}, \varphi \partial^{\alpha}\right], \varphi \partial^{\alpha}\right] u\right)_{\Omega} \\
& =(-1)^{\mu}\left(\partial_{\jmath} u,\left[-\sum_{s \in \alpha} a_{x_{s}}^{k \jmath} \partial^{\alpha-s+k}, \varphi \partial^{\alpha}\right] u\right)_{\Omega}+[*] \\
& =(-1)^{\mu}\left(\partial_{j} u, \sum_{s, l \in \alpha} \varphi \cdot a_{x_{s} x_{l}}^{k \jmath} \partial^{\alpha-s+k} \cdot \partial^{\alpha-l} u\right)_{\Omega}+[*] \\
& =-\sum_{s, l \in \alpha}\left(\partial^{\alpha-l+\jmath} u, a_{x_{s} x_{l}}^{k j} \partial^{\alpha-s+k} u\right)_{\hat{\omega}}+[*] .
\end{aligned}
$$

Here [*] are the terms which can be estimated by the quantity $C\left(\|f\|_{\mu, \Omega}^{2}+\|u\|_{\mu-1, \Omega}^{2}\right)$. Thus, when we note (2.1) and (2.6), the total contribution of $I_{3}$ in (2.7) is

$$
-\frac{1}{2} \sum_{s, l \in \alpha}\left(\partial^{\alpha-l+\jmath} u, a_{x_{s} x_{l}}^{k \jmath} \partial^{\alpha-s+k} u\right)_{\hat{\omega}}+[*] .
$$

iv) If we note $a^{k \jmath}=a^{j k}$ we have that, in (2.7), the term $I_{4}$ may only appear when $M=1, N=X_{0}$ and $M=X_{0}, N=1$. Thus when we note (2.1) and (2.6), the total contribution of $I_{4}$ in (2.7) is

$$
\begin{aligned}
\frac{1}{4}\left\{\left(A^{*} A u, X_{0} u\right)-\left(u, X_{0} A^{*} A u\right)\right\} & -\frac{1}{4}\left\{\left(X_{0} A^{*} A u, u\right)-\left(X_{0} u, A^{*} A u\right)\right\} \\
& =\frac{1}{2}\left\{\left(X_{0} u, A^{*} A u\right)-\left(u, X_{0} A^{*} A u\right)\right\}
\end{aligned}
$$




$$
\begin{aligned}
& =\frac{1}{2}\left\{\left(\left[A^{*}, X_{0}\right] A u, u\right)+\left(\left[A, X_{0}\right] u, A u\right)\right\} \\
& =\frac{1}{2}\left\{(-1)^{\mu}\left(\left[\varphi \partial^{\alpha}, b^{k} \partial_{k}\right] \varphi \partial^{\alpha} u, u\right)_{\Omega}+\left(\left[\varphi \partial^{\alpha}, b^{k} \partial_{k}\right] u, \varphi \partial^{\alpha} u\right)_{\Omega}+[*]\right. \\
& =\frac{1}{2}\left\{(-1)^{\mu} \sum_{s \in \alpha}\left(\varphi b_{x_{s}}^{k} \partial^{\alpha-s+k} \cdot \partial^{\alpha} u, u\right)_{\Omega}+\sum_{s \in \alpha}\left(\varphi b_{x_{s}}^{k} \partial^{\alpha-s+k} u, \partial^{\alpha} u\right)_{\Omega}\right\}+\left[^{*}\right] \\
& =\sum_{s \in \alpha}\left(b_{x_{s}}^{k} \partial^{\alpha-s+k} u, \partial^{\alpha} u\right)_{\hat{\omega}}+[*] .
\end{aligned}
$$

Here $\left.{ }^{*}\right]$ are the terms which can be estimated by $C\left(\|f\|_{\mu, \Omega}^{2}+\|u\|_{\mu-1, \Omega}^{2}\right)$.

Combining the above results, we have for any small $\delta>0$,

$$
\begin{aligned}
\left(-\frac{1}{2}\left(2 c-b_{x_{j}}^{\jmath}\right) \varphi \partial^{\alpha} u, \varphi \partial^{\alpha} u\right) \leqq & \delta \sum_{|\beta|=\mu}\left\|\partial^{\beta} u\right\|_{0, \hat{\omega}}^{2} \\
& +\sum_{k=1}^{n} \sum_{s \in \alpha}\left(b_{x_{s}}^{k} \partial^{\alpha-s+k} u, \partial^{\alpha} u\right)_{\hat{\omega}} \\
& +\frac{1}{2} \sum_{k, j=1}^{n} \sum_{s, l \in \alpha}\left(a_{x_{s} x_{l}}^{k j} \partial^{\alpha-l+\jmath} u, \partial^{\alpha-s+k} u\right)_{\hat{\omega}} \\
& +C\left(\|f\|_{\mu, \Omega}^{2}+\|u\|_{\mu-1, \Omega}^{2}\right),
\end{aligned}
$$

where the constant $C$ may depends on $\delta$ but is finite for each fixed $\delta>0$. Note that when $m+1 \leqq j \leqq n$ or $m+1 \leqq k \leqq n$, we can estimate the terms

$$
\delta \sum_{|\beta|=\mu}\left\|\partial^{\beta} u\right\|_{0, \hat{\omega}}^{2}, \quad\left(b_{x_{s}}^{k} \partial^{\alpha-s+k} u, \partial^{\alpha} u\right)_{\hat{\omega}}, \quad\left(a_{x_{s} x_{l}}^{k \jmath} \partial^{\alpha-l+\jmath} u, \partial^{\alpha-s+k} u\right)_{\hat{\omega}},
$$

by a quantity of the form

$$
C\left(\|f\|_{\mu, \Omega}^{2}+\|u\|_{\mu-1, \Omega}^{2}\right)+\delta \sum_{\beta=\left(\beta_{1}, \cdots, \beta_{m}, 0, \cdots, 0\right)}\left\|\partial^{\beta} u\right\|_{0, \hat{\omega}}^{2},
$$

by using (2.9) of Corollary 2.3. Thus we have the desired estimate and this proves the lemma.

\section{$\S 3$. A sufficient condition for regularity of solutions.}

In this section we shall prove the following theorem.

THEOREM 3.1. Consider the problem [P] under the conditions [A.1.], [A.2], [A.3] and [A.4]. Further we assume that (2.4) is true for some $m, 0 \leqq m \leqq n$ and for $U=\Omega$. If

$$
\left\{\begin{array}{lll}
a_{x_{k} x_{\jmath}}^{k \jmath} \leqq 0, & b_{x_{k}}^{k} \leqq 0, & k, j=1,2, \cdots, m, \\
a_{x_{s} x_{l}}^{k \jmath}=0, & b_{x_{s}}^{k}=0, & k, \jmath=1,2, \cdots, m, k \neq s, l ; j \neq s, l,
\end{array}\right.
$$


is valid on the non-elliptıc set $\omega \subset \Omega$ of $L$, then the weak solution $u \in L^{2}(\Omega)$ of $[\mathrm{P}]$ belongs to $C^{\infty}(\bar{\Omega})$.

Proof. The weak solution $u \in L^{2}(\Omega)$ of $[\mathrm{P}]$ is given by the weak limit of the solutions $u_{\varepsilon} \in C^{\infty}(\bar{\Omega})$ of $\left[\mathrm{P}_{\varepsilon}\right]$ in $L^{2}(\Omega)$. Thus, if we prove for any sufficiently small $\varepsilon>0$ and any $\mu \in N$, that

$$
\left\|u_{\varepsilon}\right\|_{\mu, \Omega}^{2} \leqq C\|f\|_{\mu, \Omega}^{2}
$$

we have

$$
\|u\|_{\mu, \Omega}^{2} \leqq \varliminf_{\varepsilon \rightarrow 0}\left\|u_{\varepsilon}\right\|_{\mu, \Omega}^{2} \leqq C\|f\|_{\mu, \Omega}^{2},
$$

and by the well known Sobolev's lemma we have $u \in C^{\infty}(\bar{\Omega})$. (see e. g. [1]).

Now we shall take and fix a function $\varphi \in C_{0}^{\infty}(\Omega)$ such that $\varphi \equiv 1$ on $\hat{\omega}, \varphi \geqq 0$ on $\Omega$, where $\hat{\omega}$ is a small neighbourhood of $\omega$ such that $\hat{\omega} \cap \partial \Omega=0$. Then we have

$$
\left\|u_{\varepsilon}\right\|_{\mu, \Omega}^{2} \leqq C\left(\left\|\varphi u_{\varepsilon}\right\|_{\mu, \Omega}^{2}+\left\|u_{\varepsilon}\right\|_{\mu, \Omega_{1} \hat{\omega}}^{2}\right),
$$

and the second term of the right can be estimated by $C\|f\|_{\mu, \Omega}^{2}$ by Lemma 2.4 . Next we shall prove that

$$
\left\|\varphi u_{\varepsilon}\right\|_{\mu, \Omega}^{2} \leqq C\left(\|f\|_{\mu, \Omega}^{2}+\left\|u_{\varepsilon}\right\|_{\mu-1, \Omega}^{2}\right) .
$$

In doing so, by Lemma 2.2 , it is sufficient to prove

$$
\sum_{\alpha}\left\|\varphi \partial^{\alpha} u_{\varepsilon}\right\|_{0, \Omega}^{2} \leqq C\left(\|f\|_{\mu, \Omega}^{2}+\left\|u_{\varepsilon}\right\|_{\mu-1, \Omega}^{2}\right)
$$

for $\alpha=\left(\alpha_{1}, \cdots, \alpha_{m}, 0, \cdots, 0\right),|\alpha|=\mu$. Now, from the assumption (3.1) and Lemma 2.5 , we have: For any small $\delta>0$, we may take a neighbourhood $\hat{\omega}$ of $\omega$ so small that

$$
\begin{array}{r}
\left(-\frac{1}{2}\left(2 c-b_{x_{k}}^{k}\right)-\left(\frac{1}{2} \sum_{k, j \in \alpha} a_{x_{k} x_{j}}^{k J}+\sum_{k \in \alpha} b_{x_{k}}^{k}\right) \partial^{\alpha} u_{\varepsilon}, \partial^{\alpha} u_{\varepsilon}\right) \hat{\omega} \\
\left.\leqq C\|f\|_{\mu, \Omega}^{2}+\left\|u_{\varepsilon}\right\|_{\mu-1, \Omega}^{2}\right)+\delta\left\|u_{\varepsilon}\right\|_{\mu, \hat{\omega}}^{2} .
\end{array}
$$

Further, we may assume that there is a positive constant $d_{0}$ such that

$$
-\frac{1}{2}\left(2 c-b_{x_{k}}^{k}\right)-\left(\frac{1}{2} \sum_{k, j \in \alpha} a_{x_{k} x_{j}}^{k j}+\sum_{k \in \alpha} b_{x_{k}}^{k}\right) \geqq d_{0}>0 \quad \text { on } \hat{\omega} .
$$

Of course it may be necessary to choose $\hat{\omega}$ small enough for each fixed $\mu$ but such $\hat{\omega}$ will exist. Thus if we take $\delta$ so small and if we take the sum of (3.2) all over $\alpha,|\alpha|=\mu$, we have

$$
d_{0} \sum_{|\alpha|=\mu}\left(\partial^{\alpha} u_{\varepsilon}, \partial^{\alpha} u_{\varepsilon}\right) \hat{\omega} \leqq C\left(\|f\|_{\mu, \Omega}^{2}+\left\|u_{\varepsilon}\right\|_{\mu-1, \Omega}^{2}\right)+\hat{\delta}\left\|u_{\varepsilon}\right\|_{\mu, \hat{\omega}}^{2},
$$

where $\hat{\delta}$ can be taken so that $d_{0}-\hat{\delta}>0$. Note that, though the constant $C$ depends 
on $\delta$ and $C \rightarrow \infty$ as $\delta \rightarrow 0$, once we fix a small $\delta>0$, then $C$ is determined as a finite positive constant. Thus we have proved that

$$
\sum_{|\alpha|=\mu}\left\|\partial^{\alpha} u_{\varepsilon}\right\|_{0, \hat{\omega}}^{2} \leqq C\left(\|f\|_{\mu, \Omega}^{2}+\left\|u_{\varepsilon}\right\|_{\mu-1, \Omega}^{2}\right)
$$

and by induction we have

$$
\sum_{|\alpha|=\mu}\left\|\partial^{\alpha} u_{\varepsilon}\right\|_{0, \Omega}^{2} \leqq C\|f\|_{\mu, \Omega}^{2},
$$

for some constant $C$ not depending on $\varepsilon$. This proves the theorem.

Remark. In the proof of the theorem, the condition [A.4] was used to get the estimate

$$
\left\|u_{\varepsilon}\right\|_{\mu, \Omega \backslash \hat{\omega}}^{2} \leqq C\|f\|_{\mu, \Omega}^{2} .
$$

Thus, if we can prove this inequality by one or other methods without supposing [A.4], the proof of the theorem runs the same. Especially, this will be possible if $L$ can be written locally by the second order ordinary differential equations in each neighbourhoods of the points in $\Omega \backslash \omega$ by taking appropriate local coordinates.

Finally we show a simple example which is an extension of the example in $\S 1$.

Example. Let $\Omega \subset \boldsymbol{R}^{n}, 0 \in \Omega$ and

$$
L u \equiv a(x) \Delta u+c(x) u=f .
$$

Suppose that i) $a(x)>0$ if $x \neq 0, a(0)=0$, ii) $c(0)<0$. Then if iii) $a_{x_{k} x_{j}}(0)=0$ for all $k, j=1,2, \cdots, n$, the weak solution of $[\mathrm{P}]$ belongs to $C^{\infty}(\bar{\Omega})$. In fact $L$ can be written in the form

$$
L u \equiv\left(a(x) u_{x_{k}}\right)_{x_{k}}-a_{x_{k}}(x) u_{x_{k}}+c(x) u .
$$

Note that from i) and iii), we can write

$$
a(x)=\sum_{|\alpha|=4} a_{\alpha} x^{\alpha}+0\left(|x|^{5}\right) .
$$

Hence it is clear that $a_{x_{k}}(0)=0$ and hence the conditions of the theorem are fulfilled.

\section{$\S 4$. Examples and comments.}

Before showing examples, we shall recall some results on the hypoellipticity of operators of second order. Suppose

$$
\begin{aligned}
L u & \equiv\left(a^{k \jmath} u_{x_{k}}\right)_{x_{\jmath}}+b^{k} u_{x_{k}}+c u \\
& =\partial_{\jmath} X_{j} u+X_{0} u+c u=f
\end{aligned}
$$


satisfies the conditions [A.1] and [A.2]. Where $X_{j}=a^{k j} \partial_{k}, j=1,2, \cdots, n, X_{0}=$ $b^{k} \partial_{k}$. Let

$$
\begin{aligned}
\mathcal{L}= & \mathcal{L}\left(X_{0}, X_{1}, \cdots, X_{n}\right) \\
= & \text { The Lie algebra with the Lie bracket }[A, B]=A B-B A \\
& \text { generated by } X_{0}, X_{1}, \cdots, X_{n} .
\end{aligned}
$$

Then it is known that:

Let $x^{0} \in \Omega$ be a point. If we can take $n$ elements

in $\mathcal{L}$ which are linearly independent at $x^{0}$,

then $L$ is hypoelliptic near $x^{0}$.

(see e.g. [8] Chap. II, §6). If $L$ satisfies [A.1], [A.2], [A.3], [A.5] and it is hypoelliptic at each points on $\bar{\Omega}$, we can assure that the weak solution belongs to $C^{\infty}(\bar{\Omega})$ without any further conditions on the coefficients of $L$. But we must distinguish the local regularity of solutions and the regularity of solutions of boundary value problems. In fact they have both similar aspects and different aspects. For example, on one hand, the condition (4.1) is related to the derivatives of the coefficients $a^{k}, b^{k}$ up to any order, on the other hand, in the known regularity theorems of the boundary value problems, the order of regularity is measured by a constant which depends only on the derivatives of $a^{k j}$, resp. $b^{k}$, up to order 2, resp. 1 . Neverthless, there seems to be a similar aspects. That is, it seems that, in the boundary value problems, the flow determined by the vector fields $X_{0}, X_{1}, \cdots, X_{n}$ have an essential role in the propagation of regularity of the solutions. As have already been pointed out in [8] in connection with the global hypoellipticity, it seems that the integral manifolds of $X_{0}, X_{1}, \cdots, X_{n}$ and the directions of $X_{0}$ (we have to distinguish positive and negative directions of $X_{0}$ ) have a certain role in the propagation of regularity (especially they will carry the regularity of the boundary data to the interior regularity of solutions).

We conjecture that the following type of results will be valid:

Assume the conditions [A.1], [A.2], [A.3] and [A.5]. Further assume that the following conditions are satisfied: For any $x^{0} \in \Omega$ we can find a curve $\mathcal{C}$ joining $x^{0}$ with some point $x^{1} \in \Sigma$ such that

i) $\mathcal{C}$ can be constructed by joining finitely many integral curves $l_{1}, \cdots, l_{s}$ of $X_{0}, X_{1}, \cdots, X_{n}$.

[C] ii) If $l_{0}$ is an integral curve of $X_{0}$, the direction of $X_{0}$ and the direction of the curve $\mathcal{C}\left(x^{1} \rightarrow x^{0}\right)$ must coincide.

iii) If there is a singular point $x^{0}$ of all the vector fields $X_{0}, X_{1}, \cdots, X_{n}$, then $x^{0}$ must be a limit point of the flow of $X_{0}$ of positive directions.

Then the weak solution $u \in L^{2}(\Omega)$ of $[\mathrm{P}]$ belongs to $C^{\infty}(\bar{\Omega})$.

Now we shall study some examples. For the simplicity we restrict our con- 
siderations to the two dimensional case.

Let

$$
\begin{gathered}
\Omega=\left\{(x, y) \in \boldsymbol{R}^{2} ; x^{2}+y^{2}<4\right\}, \quad \Omega_{1}=\left\{(x, y) \in \boldsymbol{R}^{2} ; x^{2}+y^{2}<1\right\}, \\
\Omega_{2}=\Omega \backslash \bar{\Omega}_{1} .
\end{gathered}
$$

Take and fix a function $\varphi \in C^{\infty}(\bar{\Omega})$ such that $\varphi>0$ on $\Omega_{2}, \varphi \equiv 0$ on $\bar{\Omega}_{1}$. Let $N \subset \Omega$ be the non-elliptic set of $L$.

Example 4.1. Let

$$
L u \equiv\left(\left(\varphi+x^{2 k}\right) u_{x}\right)_{x}+u_{y y}+b^{1} u_{x}+b^{2} u_{y}+c u=f .
$$

Here $X_{1}=\left(\varphi+x^{2 k}\right) \partial_{x}, X_{2}=\partial_{y}, X_{0}=b^{1} \partial_{x}+b^{2} \partial_{y}$ and $N=\{(x, y) ; x=0,|y| \leqq 1\}$. Suppose that $b^{1}, b^{2}$ and $c$ satisfy [A.3]. If $b^{1} \neq 0$ on $N$, we have that $L$ is hypoelliptic on $\bar{\Omega}$. Thus we shall restrict to the case $b^{1}=0$ on $N$. Then the sufficient conditions for $u$ to belongs to $C^{\infty}(\bar{\Omega})$ in Theorem 3.1 is

$$
\left(x^{2 k}\right)_{x x}=2 k(2 k-1) x^{2 k-2}=0, \quad b_{x}^{1} \leqq 0 \quad \text { on } N .
$$

The first condition is valid if $k>1$. Note that the second condition means that

$$
b^{1}(x, y)=-\beta(y) x+0\left(x^{2}\right), \quad \beta(y) \geqq 0 \quad \text { on } N .
$$

Further, for simplicity, consider the case when

$$
b^{1}(x, y)=b^{1}(x)=-\beta x+0\left(x^{2}\right), \quad b^{2}(x, y) \equiv 0,
$$

where $\beta$ is a positive constant. Then in terms of the flows of the vector field $X_{0}$, it can be stated that:

Near $N$, all the flows determined by $X_{0}$ flow into $N$.

Note that if $x^{0} \in \Omega \backslash N, x^{0}$ can be joined with some point $x^{1} \in \Sigma$ by an integral curve of $X_{2}=\partial_{y}$. Further, if $x^{0} \in N$, it can be joined with some $x^{1} \in \Sigma$ by an integral curve of $X_{0}$ such that the direction $x^{1} \rightarrow x^{0}$ coincides with the direction of $X_{0}$ and $x^{0}$ is a limit point of the integral curve (c.f. our conjecture [C]).

In this case, $L$ is not hypoelliptic on $N$. But by our theorem, the weak solution belongs to $C^{\infty}(\bar{\Omega})$.

Example 4.2. Let

$$
L u \equiv\left(\left(\varphi+y^{2 k}\right) u_{x}\right)_{x}+u_{y y}+b^{1} u_{x}+b^{2} u_{y}+c u=f .
$$

Here $X_{1}=\left(\varphi+y^{2 k}\right) \partial_{x}, X_{2}=\partial_{y}, X_{0}=b^{1} \partial_{x}+b^{2} \partial_{y}$ and $N=\{(x, y) ; y=0,|x| \leqq 1\}$. Since we have $\left.[\underbrace{\left[X_{2},\left[X_{2},\left[\cdots\left[X_{2}\right.\right.\right.\right.}_{2 k \text {-times }}, X_{1}] \cdots\right]=\left(\partial_{y}^{2 k} \varphi+(2 k) !\right) \partial_{x}, L$ is hypoelliptic on $\bar{\Omega}$. Hence the weak solution is in $C^{\infty}(\bar{\Omega})$. On the other hand, the condition of Theorem 3.1 
is $b_{x}^{1} \leqq 0$ on $N$. Of course it is not necessary. Note that if our conjecture [C] is true, the unnecessity of the condition $b_{x}^{1} \leqq 0$ on $N$ will follow.

Example 4.3. Let

$$
L u \equiv\left(x^{2}+y^{2}\right)^{k} u_{x x}+u_{y y}+b^{1} u_{x}+b^{2} u_{y}+c u=f .
$$

We have $N=\{(0,0)\}$. If $b^{1}(0,0)=0$, we cannot use the sufficient condition (4.1) for hypoellipticity. On the other hand, the condition of Theorem 3.1 is $b_{x}^{1}(0,0)$ $\leqq 0$, that is, $b^{1}(x, y)=-\beta(y) x+0\left(x^{2}\right), \beta(y) \geqq 0$ on $N$. For simplicity, if we consider the case when $b^{1}(x, y)=-\beta x+0\left(x^{2},|y|\right), b^{2}(x, y) \equiv 0$, in terms of the flow determined by $X_{0}$, this can be stated that:

Near the origin, all the flows of $X_{0}$ must flow into the $y$-axis.

Note that if our conjecture [C] is true, since $X_{2}=\partial_{y}$, all the weak solution $u \in L^{2}(\Omega)$ will belong to $C^{\infty}(\bar{\Omega})$ provided that the condition [A.3] is satisfied (and no other conditions concerning $b^{1}$ and $b^{2}$ will necessary).

In the same way we can treat the equation

$$
L u \equiv\left(x^{2}+y^{2}\right)^{k}\left(u_{x x}+u_{y y}\right)+b^{1} u_{x}+b^{2} u_{y}+c u=f .
$$

In this case, if $b(0,0)=0$ or $b^{2}(0,0)=0$, the sufficient condition (4.1) cannot be used. The condition of Theorem 3.1 is $b_{x}^{1} \leqq 0, b_{y}^{2} \leqq 0, b_{y}^{1}=b_{x}^{2}=0$ at $(0,0)$. Especially, this condition is satisfied if $b^{1}=-\alpha x+0\left(x^{2}+y^{2}\right), b^{2}=-\beta y+0\left(x^{2}+y^{2}\right)$ where $\alpha, \beta$ are positive constants. Thus the vector field $X_{0}$ can be apploximated by $-\alpha x \partial_{x}$ $-\beta y \partial_{y}$ and thus the above condition can be restated that:

Near the origin, all the flows of $X_{0}$ must flow into the origin.

Note that, in this case too, we can relate the above facts with the conjecture [C].

Example 4.4. Consider the equation

$$
L u \equiv \varphi \Delta u+b(x) u_{x}+c u=f .
$$

By Theorem 3.1, if $b_{x} \leqq 0$ on $\Omega_{1}$, all the weak solution is in $C^{\infty}(\bar{\Omega})$ provided that $c-b_{x} \leqq 0$ on $\Omega$. For example, this is valid if $b \equiv$ const. But it is not applicable if e. $g . b(x)=x^{2}+1$. It is quite likely that for this equation, all the weak solution is in $C^{\infty}(\bar{\Omega})$ provided that $c<0$ and $c-b_{x}<0$ on $\bar{\Omega}$.

Example 4.5. Consider the equation

$$
L u \equiv \varphi \Delta u+b^{1} u_{x}+b^{2} u_{y}+c u=f .
$$

First note that $L$ is not hypoelliptic on $\Omega_{1}$. The condition of Theorem 3.1 is

$$
b_{x}^{1} \leqq 0, b_{y}^{2} \leqq 0, b_{y}^{1}=b_{x}^{2}=0 \quad \text { on } \Omega_{1} .
$$


Now, as the simplest case of the above equation, we shall consider the following four equations :

$$
\begin{aligned}
& L_{1} u \equiv \varphi \Delta u+x u_{x}+y u_{y}+c u=f, \\
& L_{2} u \equiv \varphi \Delta u-x u_{x}-y u_{y}+c u=f, \\
& L_{3} u \equiv \varphi \Delta u-x u_{x}+y u_{y}+c u=f, \\
& L_{4} u \equiv \varphi \Delta u \pm y u_{x} \pm x u_{y}+c u=f .
\end{aligned}
$$

i) $L_{1}$ does not satisfy the condition (4.2). In this case we can assure that there are no $c_{0}>0$ such that the following statement is true:

[W] If $c \leqq-c_{0}$, all the weak solution $u \in L^{2}(\Omega)$ of [P] belongs to $C^{\infty}(\bar{\Omega})$.

In fact, let us first consider $L_{1}$ on $\Omega_{2}$. Then $\partial \Omega=\Sigma$ belongs to $\Sigma_{3}$ and $\partial \Omega_{2} \backslash \Sigma$ $=\partial \Omega_{1}$ belongs to $\Sigma_{1}$ with respect to $\Omega_{2}$ (for the definitions and properties of $\Sigma_{1}$, $\Sigma_{2}, \Sigma_{3}$, see e.g. [8] Chap I, $\S 1$ ). Thus the problem

$$
L_{1} u=f \quad \text { on } \Omega_{2},\left.\quad u\right|_{\Sigma}=0
$$

has a unique solution $u \in C^{\infty}\left(\bar{\Omega}_{2}\right)$. Since $\partial \Omega_{1}$ belongs to $\Sigma_{2}$ if we consider $L_{1}$ on $\Omega_{1}$, we have to solve the problem

$$
L_{1} \tilde{u}=f \quad \text { on } \Omega_{1},\left.\quad \tilde{u}\right|_{\partial \Omega_{1}}=g,
$$

where $g$ is defined by the solution $u$ of (4.3) by $g=\left.u\right|_{\partial \Omega_{1}}$. Now from [10], it is known that in order that, for any $g \in C^{\infty}\left(\partial \Omega_{1}\right)$, (4.4) has a unique solution in $C^{m}(\bar{\Omega})$, it is necessary and sufficient that $c(0,0)+m<0$. (More precisely, we have $\tilde{u} \in C^{\infty}\left(\Omega_{1} \backslash 0\right)$ but in general $\tilde{u}$ is not $C^{\infty}$ at the origin). Thus for $L_{1}$, the condition [W] cannot be valid for any $c$. Note that the vector field $X_{0}=x \partial_{x}+y \partial_{y}$ determines the flows which flow out from the origin.

ii) $L_{2}$ satisfies the condition of Theorem 3.1. Thus the weak solution is in $C^{\infty}(\bar{\Omega})$ provided that $c<0$ on $\bar{\Omega}$. In this case, first consider $L_{2}$ in $\Omega_{2}$. Then $\Sigma=$ $\Sigma_{3}, \partial \Omega_{1}=\Sigma_{2}$. By $[10]$,

$$
L_{2} u=f \quad \text { on } \Omega_{1}
$$

has a unique solution $u \in C^{\infty}\left(\bar{\Omega}_{1}\right)$ without any boundary conditions. Now define $\tilde{g} \in C^{\infty}\left(\partial \Omega_{1}\right)$ by $\tilde{g}=\left.u\right|_{\partial \Omega_{1}}$ and solve

$$
L_{2} u=f \quad \text { on } \Omega_{2},\left.\quad u\right|_{\Sigma}=0,\left.\quad u\right|_{\hat{o} \Omega_{1}}=\tilde{g} .
$$

Thus we have a unique $C^{\infty}$-solution of

$$
L_{2} u=f \quad \text { on } \Omega,\left.\quad u\right|_{\Sigma}=0 .
$$

Note that near the origin all the flows of $X_{0}$ flow into the origin.

iii) The operator $L_{3}$ does not satisfy the condition of Theorem 3.1, i.e. $b_{x}^{1}=$ 
$-1<0$, but $b_{y}^{2}=1>0$. In this case, the degeneracy on the $y$-axis seems to be essential and the set on which the solution is not $C^{\infty}$ is expected to coincide with $\{(x, y) ; x=0,|y| \leqq 1\}$.

iv) The operator $L_{4}$ also does not satisfy the condition of Theorem 3.1 since $b_{x}^{1}=0, b_{y}^{2}=0, b_{y}^{1}= \pm 1, b_{x}^{2}= \pm 1$. By $[10]$, it seems that since $X_{0}$ has periodic orbits, some sorts of regularity of solutions will occur. But general pictures are not clarified yet.

\section{REFERENCES}

[1] S. Agmon, Lectures on Elliptic Boundary Value Problems, Van Nostrand, 1965.

[2] M.I. Freíd In, On the Smoothness of Solutions of Degenerate Elliptic Equations, Izv. Akad. Nauk SSSR Ser. Mat. 32 (1968) ; English transl., Math. USSR-Izvestija 2 (1968), 1337-1359.

[3] A. Friedman, Stochastic Differential Equations and Applications, 2 vols., Academic Press 1975, 1976.

[4] L. Hórmander, Hypoelliptic Second Order Differential Equations, Acta Math. 119 (1967), 147-171.

[5] J. J. Kohn and L. Nirenberg, Non-Coercive Boundary Value Problems, Comm. Pure Appl. Math., 18 (1965), 443-492.

[6] J.J. Kohn and L. Nirenberg, Degenerate Elliptic-Parabolic Equations of Second Order, Comm. Pure Appl. Math., 20 (1967), 797-872.

[7] O.A. OleINIK, Linear Equations of Second Order with Nonnegative Characteristic Form, Mat. Sb., 69 (111) (1966), 111-140; English transl., Amer. Math. Soc. Transl. (2) 65 (1967), 167-199.

[8] O.A. Oleinik and E.V. Radkevic, Second Order Equations with Nonnegative Characteristic Form, Moscow, 1971; English transl., Amer Math. Soc., 1973.

[9] D. Stroock ANd S.R.S. Varadhan, On Degenerate Elliptic-Parabolic Operators of Second Order and Their Associated Diffusions, Comm. Pure Appl. Math. 25 (1972), 651-713.

[10] H. YAmAda, Boundary Value Problems for Real Linear Partial Differential Equations of First Order, Kodai Math. J., 2 (1979), 346-361.

Department of Mathematics

TOKyo Institute of TeChNOLOgY 